\title{
CorPhU: an algorithm based on phase closure for the correction of unwrapping errors in SAR interferometry
}

\author{
Angélique Benoit ${ }^{1}$, Béatrice Pinel-Puysségur ${ }^{2}$, Romain Jolivet $^{1}$ and Cécile Lasserre ${ }^{3}$ \\ ${ }^{1}$ Laboratoire de Géologie, Département de Géosciences, Ecole Normale Supérieure, PSL University, UMR CNRS 8538, Paris, France, angelique . benoi t@ens .fr. \\ 2 CEA, DAM, DIF, F-91297 Arpajon, France. \\ 3 Université de Lyon, UCBL, ENSL, CNRS, LGL-TPE, 69622 Villeurbanne, France. \\ Accepted: XXX; Received XXX; in original form XXX
}

Interferometric Synthetic Aperture Radar is commonly used in Earth Sciences to study surface displacements or construct high resolution topographic maps. Recent satellites such as those of the Sentinel-1 constellation allow to derive dense deformation maps with millimetric precision with high revisit frequency. However, InSAR is still limited by interferometric coherence. Interferometric phase noise resulting from a loss of coherence, due to changes in scattering properties between repeated SAR acquisitions, may lead to unwrapping errors, which then in turn lead to centimetric errors in time series reconstruction. We present an algorithm based on interferometric phase closure to automatically correct unwrapping errors. We describe the algorithm and highlight its performances with two case studies, in Lebanon with Envisat satellite data and in Central Turkey with Sentinel-1 data. The first dataset is particularly affected by unwrapping errors because of long spatial $(500 \mathrm{~m})$ and temporal baseline interferograms (6 years) and decorrelation due, in particular, to vegetation. The second dataset contains unwrapping errors because of temporal changes in the scattering properties of the ground. For these two examples, the algorithm allows the correction of almost all detectable unwrapping errors, without requiring visual inspection or manual deletions. Our algorithm is efficient especially on large datasets, such as with Sentinel-1 
constellation, where interferometric phase is redundant and improves eventually the reconstruction of time series.

Radar interferometry - Image processing - Creep and deformation.

\section{Introduction}

Interferometric Synthetic Aperture Radar (InSAR) is a geodetic technique developped in the 70's for geophysical applications and, originally, to construct topographic maps of the Earth (Graham, 1974; Zebker \& Goldstein, 1986), Venus (Rogers \& Ingalls, 1970) and the Moon (Zisk, 1972a,b; Margot et al., 2000). In the 90’s, InSAR was then used for the study of surface displacements related to earthquakes (Massonnet et al., 1993; Zebker et al., 1994), inflation of volcanoes (Massonnet et al., 1995) or ice sheet motion (Goldstein et al., 1993). InSAR is based on the acquisition of successive SAR images over the same area and from close positions by a side looking radar onboard a plane or a satellite. The complex conjugate product of two SAR images is called an interferogram. The phase of an interferogram, hereafter called the interferometric phase, corresponds to the relative travel time difference of the electromagnetic wave between two SAR acquisitions. The interferometric phase depends on satellite orbits, topography, spatio-temporal variations in the refractive index of the atmosphere between two acquisitions, ground deformation along the satellite line-of-sight (LOS) and various sources of noise, including Digital Elevation Model, orbits errors and instrumental noise. With two simultaneous acquisitions from two points of view, InSAR is a measurement of topography used to build DEMs, while with successive acquisitions it can be used to measure ground deformation. Measurements of deformation and ground velocity using InSAR have now reached a millimeter accuracy (Simons \& Rosen, 2015; Elliott et al., 2016).

Reconstructing continuous signals, including deformation, involves phase unwrap- 
ping, which consists in adding the appropriate multiple of $2 \pi$ to the interferometric phase and multiple methods have been developed to do so. Branch-cut algorithms consist in identifying consistent and inconsistent paths to integrate the phase signal (Goldstein et al., 1988; Prati et al., 1990; Lin et al., 1994; Herszterg et al., 2018). Least-squares techniques, weighted or unweighted, minimize the mean deviation between the estimated (wrapped) and unknown (unwrapped) discrete derivatives of the phase (Ghiglia \& Romero, 1994; Flynn, 1997; Costantini, 1998; Chen \& Zebker, 2001), sometimes using external data such as GPS to constrain the unwrapping process (Agram \& Zebker, 2010). Ultimately, Permanent or Persistent Scatterers InSAR (PS-InSAR) methods, based on the identification of pixels with stable backscattering properties in time, use the temporal information of multiple interferograms to unwrap the phase in time and space (Pepe \& Lanari, 2006; Hooper \& Zebker, 2007; Hussain et al., 2016).

However, phase unwrapping may fail, especially within low coherence regions (Rosen et al., 1996). In an interferogram, each pixel phase value corresponds to the phase of the coherent sum of backscattered electromagnetic wave from scatterers on the ground within the pixel. If scattering properties change over time or if the geometry of acquisition is too different between each pass of the satellite, the phase change between two neighbouring pixels may exceed one phase cycle (i.e. $2 \pi$ ). Coherence is a measure of the spatial correlation of phase (Lee et al., 1994). A coherence of 1 indicates the phase is constant within a cell surrounding the pixel. Over low coherence regions, higher phase noise may lead to phase differences between neighbouring pixels higher than $\pi$. In addition, large deformation gradients may lead to similar situation, for instance close to a fault that ruptured in a large seismic rupture (e.g. Simons et al., 2002).

Phase unwrapping is based on the hypothesis that the phase of two neighbouring pixels of an interferogram only differs by a fraction of $\pi$. This hypothesis is only valid in high coherence regions with a moderate fringe rate. When this assumption breaks 
down, unwrapping methods may fail, creating erroneous offsets of multiples of $2 \pi$ in the unwrapped phase. The size of the affected region may vary from a few pixels to a significant fraction of the image. In Earth science applications, almost all interferograms have large regions where phase decorrelates due to changes in scattering properties (e.g. vegetation, humidity, anthropic changes), high topographic gradients or high deformation areas and unwrapping becomes challenging (Simons et al., 2002; Zebker et al., 2007). Unwrapping errors bias estimations of surface deformation by introducing inconsistencies in the interferometric network in case of time series analysis. Unwrapping errors are sometimes manually detected and masked (e.g. Jolivet et al., 2012) and methods based on interferometric network misclosure analysis (e.g. LópezQuiroz et al., 2009) and time series analysis have been proposed (e.g. Hussain et al., 2016).

We propose an efficient algorithm, named CorPhU (CORrection of Phase Unwrapping errors), for the correction of unwrapping errors after phase unwrapping, based on the phase closure of interferogram triplets within an interferometric network. A proof of concept of this algorithm has been presented by Pinel-Puysségur et al. (2018) and we describe in details the formulation, implementation and performances of the algorithm in this paper. Phase unwrapping errors detected by the algorithm are automatically and iteratively corrected. In the following sections, we describe the algorithm and present qualitative results focusing on two case studies where decorrelation is high and could be a limiting factor, including data from the Envisat satellite over Lebanon and data from the Sentinel-1 constellation over Turkey. We then perform a quantitative assessment of the algorithm. Finally, we discuss limitations and possible improvements of our approach. 


\section{Method}

By construction, the sum of the phase of three unwrapped interferograms forming a closed loop equals 0 (Jennison, 1958). For a triplet $T$ of three SAR acquisitions $k, l$ and $m$, the triplet phase closure $\Phi_{T}$ is:

$$
\Phi_{T}=\phi_{k l}+\phi_{l m}-\phi_{k m}
$$

where $\phi_{k l}, \phi_{l m}$ and $\phi_{k m}$ are the unwrapped phase of interferograms $I_{k l}, I_{l m}$ and $I_{k m}$ computed from acquisitions $k, l$ and $m$. By construction, phase closure $\Phi_{T}$ should be equal to 0 , up to phase inconsistencies due to variations of the backscattering properties of the ground, including for instance soil moisture (De Zan et al., 2015). Once this later contribution is removed, the remaining phase closure inconsistencies correspond to phase unwrapping errors exactly equal to a multiple of $2 \pi$. Our algorithm detects and corrects such unwrapping errors within a stack of coregistred interferograms formed from SAR images (Fig. 1). First, we identify all triplets in the interferogram network. Second, we compute the phase closure for each triplet following equation 1 and identify unwrapping errors. Third, for each of these incorrectly unwrapped regions, we identify the interferogram incorrectly unwrapped among the three possible ones using the so-called "flux" or "mean closure" methods, described in sections 2.2 and 2.3 respectively. Once we have identified the interferogram incorrectly unwrapped, we correct the unwrapping error. We proceed iteratively through the network of triplets.

\subsection{Automatic identification of unwrapping errors}

For all available triplets, we start by building masks $m_{k l}, m_{l m}$ and $m_{k m}$ associated to interferograms $I_{k l}, I_{l m}$ and $I_{k m}$, based on the coherence map. Pixels with a coherence lower than a given threshold ( 0.8 by default) are masked out. If none of the three individual masks is empty, we construct the total mask of the triplet $m_{T}^{t o t}$ as the 
intersection of masks $m_{k l}, m_{l m}$ and $m_{k m}$. We then compute triplet closure on unwrapped interferograms using equation 1. We distinguish two sources of misclosure in unwrapped interferograms. The first one is unwrapping errors and is specific to unwrapped interferograms. The second one arises from interferogram multilooking prior to unwrapping. Indeed, multilooking is a non-coherent summation of different pixels, leading to small phase inconsistencies in the wrapped interferograms and thus to non-zero closure (De Zan et al., 2015). We therefore calculate the closure of wrapped interferograms, defined as:

$$
\Phi_{T}^{w}=\left(\phi_{I_{k l}}^{w}+\phi_{I_{l m}}^{w}-\phi_{I_{k m}}^{w}\right)[2 \pi]
$$

where $\phi_{k l}^{w}, \phi_{l m}^{w}$ and $\phi_{k m}^{w}$ are the phase of wrapped interferograms computed from acquisitions $k, l$ and $m$ and modulo $[2 \pi]$ indicates phase signals are within the interval $[0 ; 2 \pi]$. We substract closure of wrapped interferograms $\Phi_{T}^{w}$ from closure $\Phi_{T}$ computed on unwrapped interferograms in order to remove misclosures related to phase consistency loss in multilooking (Eq. 3, Fig. 2). The total triplet closure $\Phi_{T}^{\text {tot }}$ hence writes:

$$
\Phi_{T}^{t o t}=\left(\Phi_{T}-\Phi_{T}^{w}\right) m_{T}^{t o t}
$$

We then round the total triplet closure $\Phi_{T}^{\text {tot }}$ modulo $2 \pi$ to estimate how many multiples of $2 \pi$ should be corrected. We consider non-zero values as unwrapping errors and group them into regions using structuring elements (Fig. 3b; Verveer, 2003). Remaining zero-values (i.e. pixel has been correctly unwrapped) are grouped into regions, considered as reference for the flux method described in the next section. Phase unwrapping errors generally arise in noisy or high fringe rate areas on interferograms. The error spreads from this area, forming a connected region on which phase has been locally correctly unwrapped but is inconsistent with neighbouring regions. We then associate each unwrapping error region to the largest reference region in the vicinity. 
Now that we know where each unwrapping error is, we need to determine which interferogram of the triplet has been incorrectly unwrapped using a two-steps detection approach.

\subsection{Step 1: flux method selection}

We first try to identify which interferogram of a triplet shows an abnormal phase offset, called "flux", between an unwrapping error and its associated reference region. To compute this flux, we need to both erode and dilate the incorrectly unwrapped region to respectively isolate pixels within the unwrapping error from outside adjacent pixels, which are within a reference region (i.e. phase closure equals to zero). As explained in details in Pinel-Puysségur et al. (2018), we first fill up masked pixels within the error region (Figs 3b and c; Verveer, 2003) and we erode and dilate using a structuring element, here chosen as a square of 2x2 pixels (Matheron, 1967). The difference between the dilated and the original regions determines the outer border of the unwrapping error. Similarly, the difference between the eroded and the original regions determines the inner border of the unwrapping error (Fig. 3d). The size of the structuring element used for erosion and dilatation has been empirically chosen for the borders to be thin enough to compute the flux between neighbouring pixels but wide enough to ensure a sufficient number of flux measurements. We then discard pixels of the inner border that do not have any neighbour in the outer border, for example when they are on the image border, close to a masked region or far from the reference region. We calculate flux vectors along this boundary by differencing the phase of an inner pixel with the phase of the neighbouring outer pixel (Fig. 3e). We define $p_{\text {flux }}$ as the minimum proportion of flux vectors to correct an interferogram. For each interferogram of a triplet, we estimate the proportion of flux vectors equal to a multiple of $2 \pi$. If only one interferogram has more than $p_{\text {flux }}$ of its flux vectors equal to a multiple of $2 \pi$, this interferogram is marked as incorrectly unwrapped and the error is corrected by adding the appropriate 
multiple of $2 \pi$. If two or three interferograms have a proportion greater than $p_{\text {flux }}$, we cannot discriminate which interferogram is to be corrected. In our case, $p_{\text {flux }}$ is set to $30 \%$. This choice is empirical and based on a user decision. In our two case studies, if $p_{\text {flux }}$ is too small (i.e. we have very few flux vectors), the algorithm misses the interferogram to correct.

\subsection{Step 2: mean closure method selection}

If the flux method cannot determine which interferogram has to be corrected, we try to identify the interferogram incorrectly unwrapped by computing the mean closure of the three interferograms for all their triplets. We consider interferogram $I_{k l}$ that belongs to $N_{I_{k l}}$ triplets. The mean closure of interferogram $I_{k l}$, noted $\Phi_{I_{k l}}^{m e a n}$, is defined as the sum of the phase closure $\Phi_{n}$ on its $N_{I_{k l}}$ triplets, normalized by the number of triplets $N_{I_{k l}}$ :

$$
\Phi_{I_{k l}}^{\text {mean }}=\frac{\sum_{n=1}^{N_{I_{k l} l}} \Phi_{n}}{m_{I_{k l}}} M_{I_{k l}},
$$

where $M_{I_{k l}}$ is the intersection of all masks associated to each triplet and $m_{I_{k l}}$ is an image containing for each pixel the number of defined triplets (without mask) for interferogram $I_{k l}$. We define $p_{m c}$ as the minimum proportion of pixels equal to a multiple of $2 \pi$ to correct an interferogram. For each interferogram of the triplet, we compute the proportion of pixels in the unwrapping error zone such that the mean closure is equal to a multiple of $2 \pi$. If only one interferogram has more than $p_{m c}$ pixels equal to a multiple of $2 \pi$ in the unwrapping error region, this one is marked as incorrectly unwrapped and the error is corrected. If two interferograms fulfill this condition and if the ratio between the two proportions, noted $r_{m c}$, is greater than 2 (by default), the interferogram of highest proportion is corrected. Otherwise, we cannot discriminate which interferogram to correct. As the error may be corrected in another triplet, the algorithm then processes the following triplet. In our case studies, $p_{m c}$ is set to $50 \%$, a 
number that has been found empirically to increase the reliability of corrections. This threshold should be chosen on a case-by-case basis and is region dependent.

In general, the algorithm should be iteratively run multiple times until no unwrapping corrections are needed. Several parameters such as the size of the structuring element used for dilation and erosion and threshold values may influence the performances of the algorithm. Users have to determine which set of parameters provides adequate unwrapping error corrections for each dataset. We propose in the following to evaluate the performance of our method and provide guidelines on how to chose various parameters.

\section{A qualitative examination on two case studies}

We experiment our algorithm on two sets of SAR acquisitions and present the effects of unwrapping errors on time series analysis. First, we process the archive of SAR acquisitions from the Envisat C-Band satellite over Lebanon. There, unwrapping errors arise because of low phase coherence due to interferograms with long perpendicular baselines (max. perpendicular baseline: $500 \mathrm{~m}$; max. temporal baseline: 6 years) and to the presence of vegetation. Second, we process SAR acquisitions from the constellation of Sentinel-1 C-Band satellites over Central Turkey (max. perpendicular baseline: $250 \mathrm{~m}$; max. temporal baseline: 1 year). This constellation offers a much shorter revisit time and a larger coverage compared to products from Envisat (revisit time of 6 days, $300 \mathrm{~km}$ wide). Manual corrections of unwrapping errors cannot be performed because of the untractable size of the resulting dataset. The two case studies below differ by satellites and processing approach, however, both aim at retrieving slow tectonic deformations. As we are attempting to measure slow deformation rates at a regional scale and because of strong decorrelation effects, due for example to residual atmospheric artefacts, vegetation and snow in the winter, strong multilooking 
is required to enhance coherence in both cases, otherwise unwrapping of the phase is almost impossible.

\subsection{Application to Envisat dataset in Lebanon}

The Levant fault system is a complex active fault system of 1200 kilometers-long, where large earthquakes of magnitude up to 7.5 occurred in the past (e.g. Elias et al., 2007). This major continental fault bounds the Arabian and African plates.

We process data from Envisat ASAR track 78 with NSBAS (Doin et al., 2011), a processing chain based on the Repeat Orbit Interferometry PACkage (ROI_PAC) (Rosen et al., 2004). We coregister SLCs to a master image taking into account local topography (Guillaso et al., 2008). We use DORIS orbits from the European Space Agency (ESA) and SRTM Digital Elevation Model (DEM) Version 3.0 (Farr et al., 2007) to compute the orbital and topographic phase contributions. We multilook wrapped interferograms by a factor of 4 in range and 20 in azimuth. We use MuLSAR (Multi-Link Interferograms) in order to increase the signal-to-noise ratio of interferograms (Pinel-Puysségur et al., 2012). We then correct wrapped interferograms from stratified tropospheric delays estimated from the ERA-Interim global atmospheric reanalysis from ECMWF (Doin et al., 2009; Jolivet et al., 2011). We evaluate and compensate DEM errors by estimating the bias induced by perpendicular baselines (Ducret et al., 2014). Finally, we filter interferograms using a Goldstein filter (Goldstein \& Werner, 1998), multilook by an additional factor of 4 (16 looks in range, 80 looks in azimuth) and unwrap them using the branch-cut method (Goldstein et al., 1988). Our final dataset is made of 165 unwrapped interferograms.

Our algorithm identifies 282 triplets, among which 186 are corrected. We illustrate automatic corrections with a long temporal baseline interferogram, spanning 4 years, where three corrections are performed (Fig. 4). The first error (number 1 in Fig. 4) is clearly well corrected. The two other errors (number 2 and 3 in Fig. 4) are more 
challenging due to the effect of filtering on high fringe rate areas. In both cases, a sharp fringe, partially visible on the interferogram before filtering (arrows in Fig. 4d and $\mathrm{f}$ ), disappears through filtering (arrows in Fig. 4e and g) hence leading to discontinuities in the unwrapped interferograms (red circles in Fig. 4b). After correction, the algorithm restores continuity where a $2 \pi$ phase offset was inconsistently introduced by the unwrapping procedure (red circles in Fig. 4c) and discontinuity in high fringe rate areas (arrows in Fig. 4c).

\subsection{Application to Sentinel-1 dataset in Central Turkey}

The North Anatolian Fault is an active right-lateral strike-slip fault that accommodates the rotation of Anatolia with respect to Eurasia. During the $19^{\text {th }}$ century, seismic activity was characterized by a westward propagation of large earthquakes $\left(\sim \mathrm{M}_{\mathrm{W}} 7.0\right)$ along this 1200 kilometers-long fault (Stein et al., 1997). The last earthquake to date is the Izmit event $M_{W} 7.5$ in 1999, east of the Sea of Marmara (e.g. Reilinger et al., 2000).

We process data from Sentinel-1 track 87 with the InSAR Scientific Computing Environment (ISCE) software (Gurrola et al., 2010). We define the acquisition of July, $9^{\text {th }}$ 2017 as the master Single Look Complex (SLC) and coregister all SLCs to this master image. Coregistration is enhanced using the spectral diversity of burst overlaps refined within the network of interferograms (Fattahi et al., 2017). We generate interferograms, accounting for digital elevation model (SRTM Version 3.0; Farr et al., 2007) and orbital contributions, and merge tiles for each of them using bursts and swaths overlaps. We multilook merged interferograms with factors of respectively 81 and 27 in azimuth and range directions for a final pixel size of 540 x 420 meters, in range and azimuth respectively. We correct the phase from tropospheric signals using ERA-5, the latest global atmospheric reanalysis from ECMWF (Hersbach \& Dee, 2016). Finally, we filter (Goldstein \& Werner, 1998) and unwrap interferograms using the branch-cut 
method (Goldstein et al., 1988). Before building triplets, we discard low coherence interferograms which cannot be sufficiently unwrapped (less than $20 \%$ of the area). Our final dataset is made of 686 coregistered and unwrapped interferograms.

Our algorithm identifies 2880 triplets, among which 986 triplets are corrected eventually (Fig. 5a). We calculate the percentage of corrected pixels per interferogram by summing the number of pixels detected as unwrapping errors and corrected by the algorithm in all triplets of the interferogram. We see that most of the interferograms are totally corrected from unwrapping errors during a first pass of the algorithm (Fig. 5b). We illustrate automatic corrections with two examples of corrected interferograms, one with a large unwrapping error of 10388 pixels (4\% of the interferogram, Fig. 5c) and another with two unwrapping errors localized in different places (Fig. 5d). In both cases, $99 \%$ of the unwrapping error is automatically detected and corrected by the algorithm. Uncorrected pixels are located in the masked region of the triplet. The second example shows that the algorithm can perform multiple corrections in a single interferogram (Fig. 5d). In this case, it detects two unwrapping errors in the same interferogram and corrects them in the same triplet.

\section{Discussion and quantitative tests}

\subsection{Unwrapping errors and time series analysis}

One potential application of SAR interferometry is to perform time series analysis and estimate ground velocity over a given region from a stack of interferograms. We illustrate the effect of automatic corrections of unwrapping errors on the estimation of ground velocity and the associated decrease in errors on ground surface deformation measurement.

We perform two time series analysis on the Sentinel-1 dataset (Section 3.2): the first one is applied to the original stack of interferograms not corrected from unwrap- 
ping errors, the second one is applied to the interferograms corrected by the proposed approach. We invert the temporal evolution of the phase for both datasets identically using the small baseline NSBAS approach (Doin et al., 2011) implemented in the Generic InSAR Analysis Toolbox (GIAnT) (Agram et al., 2012). In this method, we consider each pixel independently to recover the phase change with time (López-Quiroz et al., 2009; Doin et al., 2011; Jolivet et al., 2012). In addition to phase reconstruction, NSBAS includes a time dependent model of the phase to predict the phase evolution with time when interferometric links are missing between two disconnected subsets of interferograms.

For each time series analysis, we first remove interferograms that have less than $35 \%$ unwrapped pixels, hence reducing the dataset to 627 interferograms. We then multilook interferograms by a factor of 2 in order to reduce noise on the interferograms (due to the presence of vegetation and snow) and spatially reference them by choosing a region where the phase is set to be equal in all interferograms. We correct orbital biases in interferograms by estimating a linear ramp. Terms of the ramp are refined accounting for the interferometric network (Lin et al., 2010; Jolivet et al., 2012). We then perform a least squares inversion of phase delays of each pixel to solve for the total phase delay of each date relative to the first date and for a parametric evolution of phase change across the whole acquisition period. The parametric evolution of surface deformation is a combination of a linear term and a seasonal-annual function.

We obtain two velocity maps over Central Turkey (Fig. 6a and b). If we do not correct interferograms from unwrapping errors before the inversion, surface velocity is strongly affected by unwrapping errors (Fig. 6a, b, d and e). In particular, several suspicious discontinuities visible on the first velocity map (Fig 6a and 6d) are not detected on the second one (Fig $6 \mathrm{~b}$ and 6e). The difference in velocity between the two fields reaches up to $4 \mathrm{~mm} / \mathrm{yr}$ in large regions (Fig. 6c), corresponding in our case to about $20 \%$ of the expected tectonic displacement in the area. We can also identify small dif- 
ferences of about $1 \mathrm{~mm} / \mathrm{yr}$ (Fig. 6c and $\mathrm{f}$ ), due to a difference in referencing between the two velocity maps. If we choose a reference region within an unwrapping error, the inversion will differ hence the resulting velocity maps will be different.

The effect of unwrapping errors can be evaluated quantitatively by computing a Root Mean Square (RMS) map, defined as:

$$
\Phi_{R M S}=\frac{1}{N}\left[\sum_{N}\left(\phi_{i j}-\sum_{k=i}^{j-1} m_{k}\right)^{2}\right]^{1 / 2}
$$

where $\phi_{i j}$ is the measured phase between acquisitions $i$ and $j$ and $\sum_{k=i}^{j-1} m_{k}$ is the reconstructed phase between the same acquisitions (Fig. 7; Cavalié et al., 2007). This RMS evaluates the quality of the time series reconstruction and should then reflect interferometric misclosure. If we do not correct interferograms from unwrapping errors before the inversion, RMS reaches $12 \mathrm{~mm}$ (Fig. 7a), compared to few millimeters if unwrapping errors are corrected with the proposed approach (Fig. 7c). Average RMS is of $1.61 \mathrm{~mm}$ without corrections and $0.98 \mathrm{~mm}$ with corrections. In the case where unwrapping errors are not corrected, deviation in RMS is much larger than when errors are corrected, with extreme values of 8 to $14 \mathrm{~mm}$ (Fig. $7 \mathrm{~b}$ ). Since pixels with a large RMS after time series analysis cannot be trusted for further interpretation, our approach allows to extend the area over which we can interpret the LOS displacement signal. Therefore, correcting unwrapping errors allows to expand the zone over which we confidently measure ground velocity, in the present case by $20 \%$ with a RMS threshold of $3 \mathrm{~mm}$.

\subsection{Parametric tests on the Lebanon dataset}

The validation of an automatic correction algorithm for phase unwrapping errors is a difficult task. The ideal way to assess the performance of such an algorithm would be to have a comprehensive ground truth where every pixel of every interferogram of 
the database is labelled as correctly or incorrectly unwrapped. Then, for each pixel incorrectly unwrapped, the number of cycles of the error and its associated sign should be known. In addition, such ground truth would allow identifying false alarms, i.e. pixels correctly unwrapped but detected as incorrectly unwrapped.

Practically, on a real database, there is no simple and efficient way to determine which pixels have been incorrectly unwrapped. It should be stressed that pixels incorrectly unwrapped may be detected easily on a triplet thanks to its closure but that determining them directly on interferograms is a tedious task. Indeed, even a thorough visual examination of interferograms does not always allow determining if a region is incorrectly unwrapped and if so to which extent. Furthermore, no independent dataset provides comparable measurement at the resolution allowed by InSAR.

There are two strategies to quantitatively validate the results of the proposed algorithm. The first one is to establish an experimental ground truth on a part of a real database by visual inspection. The second one would be to create a synthetic database on which unwrapping errors would be known. This last one will be explored in future work. In this paper, we chose to derive quantitative performances attempting to compare with our experimental ground truth.

Because we need to identify manually unwrapping errors, we will apply our validation on the Lebanon dataset. We select 22 interferograms with easily identifiable unwrapping errors. For each interferogram, we manually detect and label the regions of unwrapping errors. For each region, we also identify the signed number of phase cycles of the error. We thoroughly perform this manual task to ensure that the experimental ground truth does not contain any error. The ground truth contains 26 error regions with a total of 120235 pixels. To check if the algorithm detects false alarms, we select 15 interferograms and manually cut out and store regions that do not contain any unwrapping errors, with a total of 983310 pixels.

CorPhU depends on four parameters (see Table 1). Instead of a full parametric 
study which is beyond the scope of this paper, we focus our parametric tests on two out of the four parameters identified as the most important ones, including the thresholds $p_{f l u x}$ and $p_{m c}$ used for the identification of the incorrectly unwrapped interferogram of a triplet.

$p_{f l u x}$ and $p_{m c}$, two proportions of flux or pixels, are defined between 0 and $100 \%$. For the parametric tests, we test values ranging from $10 \%$ and $90 \%$ with a step of $20 \%$. We also test the algorithm with only one of the two steps. To do so, we set $p_{f l u x}$ (resp. $p_{m c}$ ) to a value strictly greater than $100 \%$. As a proportion would never attain such a value, we only use the $2^{\text {nd }}$ step (resp. $1^{\text {st }}$ step) during the run. In practice, the case with both $p_{f l u x}$ and $p_{m c}$ strictly greater than $100 \%$ prohibits any correction and is not applicable.

We run CorPhU with each of these combinations on the whole Lebanon dataset, with two iterations. We then compute the True Detection (TD) and False Alarm (FA) rates on the ground truth as follows. For each region labelled as incorrectly unwrapped in the ground truth, we compute the difference between the interferogram obtained after CorPhU's processing and the original one. We compare this difference to the expected correction, which is known from our estimates of ground truth. If these quantities are equal, then the error region has been well detected. Then, we compute the ratio of well detected regions as the TD rate. For the FA rate, we compare all regions of the ground truth labelled as correctly unwrapped to the difference of the interferograms before and after CorPhU's processing. This difference should be zero. If not, we count the region as a FA.

The results do not show any sufficient variation as a function of the parameters. For almost every test, the TD rate is quasi constant between $46 \%$ and $50 \%$. The only cases where the TD rate decreases significantly (under $40 \%$ ) are for $p_{m c}$ or $p_{\text {flux }}$ strictly greater than $100 \%$ (only one of the steps is used). Similarly, the FA rate is almost always equal to 0 except for some cases where it reaches $2 \%$. These results do not 
sufficiently vary to draw any conclusion on the influence of the parameters. However, we can draw two conclusions.

First, only half of unwrapping errors of this particular ground truth are detected. This poor performance can be explained as follows. In the Lebanon test case, there are only 282 triplets for 165 interferograms or an average of 1.71 triplets per interferogram. Moreover, many interferograms are partially masked. As triplet closure is only defined on the intersection of the definition domain of the three interferograms, the effective number of triplets per interferogram is even strictly smaller than 1.71. CorPhU should work better when the number of triplets increases for several reasons: first, the errors can only be detected on triplets closures; second, the first step needs a neighboring reference region on which the triplet closure is defined; third, the second step needs at least two triplets per interferogram under examination to determine which one is badly unwrapped. The poor TD rate on this database could thus be explained by the lack of triplets and the large masked areas, especially compared to the Turkey dataset where an interferogram belongs to 8.2 triplets on average.

Second, we think our estimate of ground truth is not sufficient and not precise enough to assess the parameters influence on CorPhU's performances. It highlights the need of a synthetic database where the number of interferograms and triplets, the masked areas surface or other variables could be changed and their effect on CorPhU's performances properly assessed.

Consequently, we choose to assess the influence of tunable parameters on the performance by counting the number of pixels incorrectly unwrapped before and after the run. We automatically compute the total number of pixels on all triplets with non-zero triplet closure, $W$. We then derive the difference $\Delta W$ between $W$ before $\left(W_{\text {init }}\right)$ and after $\left(W_{\text {final }}\right)$ the run to derive the percentage of corrected pixels (here, $W_{\text {init }}$ is equal to 1847173 pixels, see Table 2) .

Table 2 shows that the percentage of corrected pixels highly depends on the pa- 
rameter value. We highlight in green in Table 2 the range of best parameters. We highlight acceptable sets of parameters in blue while red values correspond to settings that should not be used. Settings highlighted in green allow more than $90 \%$ of the detected pixels to be corrected. The algorithm performs the best when $p_{\text {flux }}=50 \%$, whatever the value of $p_{m c}$. However, for $p_{f l u x}<=30 \%$, the performances slightly deteriorate for decreasing $p_{\text {flux }}$. Although still very acceptable for $p_{\text {flux }}=30 \%$, the performances are less good for $p_{f l u x}=10 \%$, especially if $p_{m c}$ is small. Surprisingly, for $p_{\text {flux }}$ between $10 \%$ and $50 \%$, CorPhU still performs well even without the second step (see last line of Table 2).

For $p_{\text {flux }}>=70 \%$, performances degrade with increasing $p_{\text {flux }}$. In particular, for $p_{\text {flux }}>1$ (only step 2), the percentage of corrected pixels drops under $35 \%$. When $p_{\text {flux }}$ increases, less corrections are possible with step 1 so CorPhU moves to step 2 to determine the wrong interferogram. The loss of performance suggests that step 2 is less effective than step 1 and that relying only on step 2 degrades the performances of CorPhU in our case. This suggests that many efficient corrections due to step 1 disappear when $p_{f l u x}$ is too high. It should be noted however that the poor performance of the second step in the Lebanon case may be due to the relatively small number of triplets as a greater number of triplets should enhance the robustness of the second step. In general, if $p_{f l u x}$ is set between $10 \%$ and $50 \%$ and $p_{m c}$ between $30 \%$ and $90 \%$, at least $85 \%$ of the pixels are corrected.

Nonetheless, analysis of this difference might not be a perfect assessment method for several reasons. First, some unwrapping errors cannot be detected by triplet misclosure, because the interferogram does not belong to any triplet or because of the masked areas. Second, errors cannot be detected either when two interferograms of the same triplet contain on a common region errors that compensate each other in the closure. This is the case when one acquisition of the triplet contains a sharp atmospheric delay incorrectly unwrapped in two interferograms. Third, the true parameter of interest 
would be the total number of incorrectly unwrapped pixels on all interferograms. Instead, $W$ counts several times the same error of an interferogram as soon as it belongs to several triplets. Thus there is no simple relationship between these two variables. Fourth, if the misclosure of a triplet disappears after the run, it is hopefully due to a right correction but it also may be due to an incorrect correction. Although such a wrong correction may then increase the number of pixels with non-zero closure on other triplets, it is not always the case. Nonetheless, $W$ seems to vary for a given range of parameters and tendances arises, hence it is a proxy of the performance of CorPhU.

Even if this parametric analysis is partial, some conclusions can be drawn: the most determining factor is $p_{\text {flux }}$ which should be set ideally around $50 \%$ whereas $p_{m c}$ should lie above $30 \%$. However, these results are relative to a specific dataset, hence they should be taken with caution if applied to another dataset. Among others, the number of interferograms and triplets, the multilooking factors, the shape and size of decorrelating areas should influence the optimal choice of parameters. A solution to determine the best set of parameters for a given dataset would be to first run $\mathrm{CorPhU}$ for different sets of parameters in order to make an automatic diagnosis. The algorithm should then be run again with appropriate values of parameters. Another solution would be to vary the parameters settings during the iterations. The thresholds could be set to high values during the first iterations to allow only few corrections with a high confidence level and progressively decrease during the following iterations. The aim is to avoid any false corrections at the beginning of the process that may later induce other false corrections.

\subsection{Effectiveness of the algorithm in correcting unwrapping errors in high redundancy datasets}

We also assess the effectiveness of the algorithm in correcting unwrapping errors in large datasets using the proxy $W$, corresponding to the total number of pixels on all 
triplets with non-zero closure (unwrapping errors) automatically computed before and after two successive runs. We run the algorithm four times, iteratively, on the 686 interferograms of the Turkey dataset. Before performing any corrections, the algorithm detects around 2 millions of pixels incorrectly unwrapped on the 2880 triplets built (Figure 8). At the end of the first run, the number of pixels corresponding to unwrapping errors is about 500000 , indicating that the algorithm corrects $75 \%$ of the initial pixels in a single iteration. At the end of the second run, the number of pixels decreases to 100000 , illustrating that the algorithm corrects $95 \%$ of the initial incorrectly unwrapped pixels in only two iterations. Next runs show that the algorithm converge in two iterations, as the number of pixels incorrectly unwrapped does not decrease anymore after the second run. This might be due to the size of residual errors, too small to be corrected considering our threshold, for this particular case, of a minimum 200 pixels for a single unwrapping error, or to errors which are not or no longer connected to references regions (no misclosure), and therefore where the flux method cannot be performed. As a conclusion, the strength of this algorithm is that it automatically corrects almost all of the unwrapping errors from a large dataset in only two iterations.

\subsection{Effectiveness of the algorithm in correcting unwrapping errors using a least-square unwrapping method}

We test our algorithm not only on interferograms unwrapped using a branch-cut method but also on those unwrapped using a least-square approach. We unwrap the 686 interferograms of the Turkey dataset using the Snaphu algorithm and run the algorithm four times as described above. Before performing any corrections, the algorithm detects around 20 millions of pixels incorrectly unwrapped on the 2880 triplets built, compared to the 2 millions of pixels for the branch-cut method (Figure 9). At the end of the first run, the number of pixels corresponding to unwrapping errors does not decrease significantly, as only 20000 pixels have been corrected during the iteration. Other iter- 
ations do not lead to perform a high number of corrections.

Results suggest that the algorithm is not adapted for interferograms unwrapped using a least-square approach, such as Snaphu. First, as Snaphu is a global minimization procedure, resulting triplets phase closure maps are not obvious to interpret, compared to those computed for interferograms unwrapped using a branch-cut method. Closure maps are equal to 0 plus or minus a residual that might not necessarily equal a multiple of $2 \pi$. Therefore, the algorithm detects, in this case, ten times more pixels incorrectly unwrapped with Snaphu than with the branch-cut method. Second, global minimization leads to a less marked spatial signature of unwrapping errors (lower phase gradients between outer and inner pixels of an unwrapping error). Consequently, the so-called flux method of our algorithm, designed for the detection of unwrapping errors using phase gradients, fails most of the time. The mean closure method also fails most of the time in correcting unwrapping errors because errors are not necessarily a multiple of $2 \pi$. Further work is required to adapt our method to global minimization unwrapping methods.

\section{Conclusions, limits and future work}

We developed an algorithm called CorPhU, using phase closure of triplets of interferograms to correct unwrapping errors left after phase unwrapping. We assess its efficiency on two datasets in Lebanon and Turkey, respectively with Envisat and Sentinel-1 satellites. Our algorithm helps the interpretation of the interferometric phase in low coherence regions, polluted by unwrapping errors, without requiring visual interferogram inspection or manual deletions of unwrapping errors. As the contribution of unwrapping errors to velocity maps may reach up to $1 \mathrm{~cm} / \mathrm{yr}$ and as they lead to RMS errors up to $1 \mathrm{~cm}$, it is critical to correct these errors for interseismic strain measurements in active tectonic environments, where deformation rates are typically on the order of millimeters per year. 
As the algorithm is based on triplet information, the more interferograms are constructed, the largest the network of triplets is built, hence the higher the probability to correct recurrent unwrapping errors. The algorithm is particularly powerful for large datasets such as from Sentinel-1, where the revisit time is 6 days hence allowing to construct large networks. However, there are some limitations. Processing time is one of the main constraints and depends on the size of the dataset. For example, the algorithm takes about six hours to process the Turkey dataset, which corresponds to 2880 triplets, using 24 threads on a classic desktop machine. One way to increase the speed of processing is to take more benefits from triplets information considering the first iteration. The goal is to determine which interferograms to correct first so that it helps for the correction of other interferograms, hence reducing processing time. For instance, triplets with small-baseline interferograms should be corrected in priority as they are supposed to be less affected by decorrelation and therefore less affected by unwrapping errors. Long-baseline interferograms should be corrected afterwards, using triplets where small-baseline interferograms have been corrected. Another improvement would be to parallelize some of the steps of the algorithm, for instance to deal with independent triplets in parallel.

Our automatic method, designed for dense networks of interferograms, requires technical improvements but, in overall, fits well into existing lines of research, where we increasingly face "big data" related challenges, which must be converted from a highway to hell to a stairway to heaven.

\section{Acknowledgments}

This project received fundings from the European Research Council (ERC) under the European Union's Horizon 2020 research and innovation program (Geo-4D project, grant agreement 758210). We use the Sentinel-1 and Envisat products, respectively provided by the Plateforme d'Exploitation des Produits Sentinel (PEPS) for Turkey 
and ESA through Cat1 proposal and EOLi-SA platform for Lebanon. We process acquisitions using the ISCE system developed at JPL/Caltech (Turkey) and NSBAS chain based on ROI_PAC as well (Lebanon). This work was partially supported by The Laboratoire de Recherche Commun Yves Rocard. Data analysis on Lebanon was supported by CNES through the TOSCA program. The code of our open-source algorithm is available on Github (https://github.com/AngeliqueBenoit/CorPhU).

\section{References}

Agram, P., Jolivet, R., \& Simons, M., 2012. Generic InSAR Analysis Toolbox (GIAnT) - User Guide, http://earthdef.caltech.edu.

Agram, P. S. \& Zebker, H., 2010. Edgelist phase unwrapping algorithm for time series InSAR analysis, J. Opt. Soc. Am. A, 27(3).

Cavalié, O., Doin, M.-P., Lasserre, C., \& Briole, P., 2007. Ground motion measurement in the Lake Mead area, Nevada, by differential synthetic aperture radar interferometry time series analysis: Probing the lithosphere rheological structure, J. Geophys. Res., 112(B3).

Chen, C. W. \& Zebker, H. A., 2001. Two-dimensional phase unwrapping with use of statistical models for cost functions in nonlinear optimization, J. Opt. Soc. Am. A, 18(2), 338-351.

Costantini, M., 1998. A novel phase unwrapping method based on network programming, IEEE Trans. Geosci. Remote Sens., 36(3), 813-821.

De Zan, F., Zonno, M., \& Lopez-Dekker, P., 2015. Phase Inconsistencies and Multiple Scattering in SAR Interferometry, IEEE Trans. Geosci. Remote Sens., 53(12), 66086616.

Doin, M.-P., Lasserre, C., Peltzer, G., Cavalié, O., \& Doubre, C., 2009. Corrections of stratified tropospheric delays in SAR interferometry: Validation with global atmospheric models, J. Appl. Geophys., 69(1), 35-50.

Doin, M.-P., Lodge, F., Guillaso, S., Jolivet, R., Lasserre, C., Ducret, G., Grandin, R., Pathier, E., \& Pinel, V., 2011. Presentation of the small baseline NSBAS processing chain on a case example: the Etna deformation monitoring from 2003 to 2010 using Envisat data, Proc. ESA 'Fringe 2011 Work. Frascati, Italy, (19-23 Sept. 2011), pp. $19-23$.

Ducret, G., Doin, M.-P., Grandin, R., Lasserre, C., \& Guillaso, S., 2014. DEM Corrections Before Unwrapping in a Small Baseline Strategy for InSAR Time Series Analysis, IEEE Geosci. Remote Sens. Lett., 11(3), 696-700. 
Elias, A., Tapponnier, P., Singh, S. C., King, G. C., Briais, A., Daëron, M., Carton, H., Sursock, A., Jacques, E., Jomaa, R., \& Klinger, Y., 2007. Active thrusting offshore Mount Lebanon: Source of the tsunamigenic A.D. 551 Beirut-Tripoli earthquake, Geology, 35(8).

Elliott, J., Walters, R., \& Wright, T., 2016. The role of space-based observation in understanding and responding to active tectonics and earthquakes, Nat. Commun., 7(1).

Farr, T. G., Rosen, P. A., Caro, E., Crippen, R., Duren, R., Hensley, S., Kobrick, M., Paller, M., Rodriguez, E., Roth, L., Seal, D., Shaffer, S., Shimada, J., Umland, J., Werner, M., Oskin, M., Burbank, D., \& Alsdorf, D., 2007. The Shuttle Radar Topography Mission, Rev. Geophys., 45(2).

Fattahi, H., Agram, P., \& Simons, M., 2017. A Network-Based Enhanced Spectral Diversity Approach for TOPS Time-Series Analysis, IEEE Trans. Geosci. Remote Sens., 55(2), 777-786.

Flynn, T. J., 1997. Two-dimensional phase unwrapping with minimum weighted discontinuity, J. Opt. Soc. Am. A, 14(10).

Ghiglia, D. C. \& Romero, L. A., 1994. Robust two-dimensional weighted and unweighted phase unwrapping that uses fast transforms and iterative methods, J. Opt. Soc. Am. A, 11(1).

Goldstein, R. M. \& Werner, C. L., 1998. Radar interferogram filtering for geophysical applications, Geophys. Res. Lett., 25(21), 4035-4038.

Goldstein, R. M., Zebker, H. A., \& Werner, C. L., 1988. Satellite radar interferometry: Two-dimensional phase unwrapping, Radio Sci., 23(4), 713-720.

Goldstein, R. M., Engelhardt, H., Kamb, B., \& Frolich, R. M., 1993. Satellite Radar Interferometry for Monitoring Ice Sheet Motion: Application to an Antarctic Ice Stream, Science (80-. )., 262(5139), 1525-1530.

Graham, L., 1974. Synthetic interferometer radar for topographic mapping, Proc. IEEE, 62(6), 763-768.

Guillaso, S., Lasserre, C., Doin, M.-P., Cavalie, O., Sun, J., \& Pelzer, G., 2008. InSAR measurement of interseismic strain in areas of low coherence: example across the Haiyuan fault (Gansu, China) using a local InSAR adaptive range filter, $E G U, \mathbf{1 0}$.

Gurrola, E., Rosen, P. A., Sacco, G. F., Seliga, W., Zebker, H., Simons, M., \& Sandwell, D., 2010. InSAR Scientific Computing Environment, 2010 Am. Geophys. Union Meet.

Hersbach, H. \& Dee, D., 2016. ERA5 reanalysis is in production, ECMWF Newsl., 147.

Herszterg, I., Poggi, M., \& Vidal, T., 2018. 2D-Phase Unwrapping via Balanced Spanning Forests, (1995). 
Hooper, A. \& Zebker, H. A., 2007. Phase unwrapping in three dimensions with application to InSAR time series, J. Opt. Soc. Am. A, 24(9).

Hussain, E., Hooper, A., Wright, T. J., Walters, R. J., \& Bekaert, D. P. S., 2016. Interseismic strain accumulation across the central North Anatolian Fault from iteratively unwrapped InSAR measurements, J. Geophys. Res. Solid Earth, 121(12), 9000-9019.

Jennison, R. C., 1958. A Phase Sensitive Interferometer Technique for the Measurement of the Fourier Transforms of Spatial Brightness Distributions of Small Angular Extent, Mon. Not. R. Astron. Soc., 118(3), 276-284.

Jolivet, R., Grandin, R., Lasserre, C., Doin, M.-P., \& Peltzer, G., 2011. Systematic InSAR tropospheric phase delay corrections from global meteorological reanalysis data, Geophys. Res. Lett., 38(17).

Jolivet, R., Lasserre, C., Doin, M.-P., Guillaso, S., Peltzer, G., Dailu, R., Sun, J., Shen, Z.-K., \& Xu, X., 2012. Shallow creep on the Haiyuan Fault (Gansu, China) revealed by SAR Interferometry, J. Geophys. Res. Solid Earth, 117(B6).

Lee, J., Hoppel, K., Mango, S., \& Miller, A., 1994. Intensity and phase statistics of multilook polarimetric and interferometric SAR imagery, IEEE Trans. Geosci. Remote Sens., 32(4), 1017-1028.

Lin, Q., Vesecky, J. F., \& Zebker, H. A., 1994. Phase unwrapping through fringe-line detection in synthetic aperture radar interferometry, Appl. Opt., 33(2).

Lin, Y.-n. N., Simons, M., Hetland, E. A., Muse, P., \& DiCaprio, C., 2010. A multiscale approach to estimating topographically correlated propagation delays in radar interferograms, Geochem., Geophys. Geosys., 11(9).

López-Quiroz, P., Doin, M.-p., Tupin, F., Briole, P., \& Nicolas, J.-m., 2009. Time series analysis of Mexico City subsidence constrained by radar interferometry, $J$. Appl. Geophys., 69(1), 1-15.

Margot, J.-L., Campbell, D., Jurgens, R., \& Slade, M., 2000. Digital elevation models of the Moon from Earth-based radar interferometry, IEEE Trans. Geosci. Remote Sens., 38(2), 1122-1133.

Massonnet, D., Rossi, M., Carmona, C., Adragna, F., Peltzer, G., Feigl, K., \& Rabaute, T., 1993. The displacement field of the Landers earthquake mapped by radar interferometry, Nature, 364(6433), 138-142.

Massonnet, D., Briole, P., \& Arnaud, A., 1995. Deflation of Mount Etna monitored by spaceborne radar interferometry, Nature, 375(6532), 567-570.

Matheron, G., 1967. Elements pour une theorie des milieux poreux, Paris, masson edn.

Pepe, A. \& Lanari, R., 2006. On the Extension of the Minimum Cost Flow Algorithm for Phase Unwrapping of Multitemporal Differential SAR Interferograms, IEEE Trans. Geosci. Remote Sens., 44(9), 2374-2383. 
Pinel-Puysségur, B., Michel, R., \& Avouac, J.-P., 2012. Multi-Link InSAR Time Series: Enhancement of a Wrapped Interferometric Database, IEEE J. Sel. Top. Appl. Earth Obs. Remote Sens., 5(3), 784-794.

Pinel-Puysségur, B., Lasserre, C., Benoit, A., Jolivet, R., Doin, M.-p., \& Champenois, J., 2018. A Simple Phase Unwrapping Errors Correction Algorithm Based on Phase Closure Analysis, in IGARSS 2018 - 2018 IEEE Int. Geosci. Remote Sens. Symp., vol. 1, pp. 2212-2215, IEEE.

Prati, C., Giani, M., \& Leuratti, N., 1990. SAR Interferometry: A 2-D Phase Unwrapping Technique Based On Phase And Absolute Values Informations, in 10th Annu. Int. Symp. Geosci. Remote Sens., pp. 2043-2046, IEEE.

Reilinger, R. E., Ergintav, S., Bürgmann, R., McClusky, S., Lenk, O., Barka, A., Gurkan, O., Hearn, L., Feigl, K. L., Cakmak, R., Aktug, B., Ozener, H., \& Töksoz, M. N., 2000. Coseismic and Postseismic Fault Slip for the 17 August 1999, M = 7.5, Izmit, Turkey Earthquake, Science (80-. )., 289(5484), 1519-1524.

Rogers, A. E. E. \& Ingalls, R. P., 1970. Radar Mapping of Venus With Interferometric Resolution of the Range-Doppler Ambiguity, Radio Sci., 5(2), 425-433.

Rosen, P. A., Hensley, S., Zebker, H. A., Webb, F. H., \& Fielding, E. J., 1996. Surface deformation and coherence measurements of Kilauea Volcano, Hawaii, from SIR-C radar interferometry, J. Geophys. Res., 101(E10).

Rosen, P. A., Hensley, S., Peltzer, G., \& Simons, M., 2004. Updated repeat orbit interferometry package released, Eos, Trans. Am. Geophys. Union, 85(5).

Simons, M. \& Rosen, P., 2015. Interferometric Synthetic Aperture Radar Geodesy, in Treatise Geophys., vol. 3, pp. 339-385, Elsevier.

Simons, M., Fialko, Y., \& Riviera, L., 2002. Coseismic Deformation from the 1999 Mw 7.1 Hector Mine, California, Earthquake as Inferred from InSAR and GPS Observations, Bull. Seismol. Soc. Am., 92(4), 1390-1402.

Stein, R. S., Barka, A. A., \& Dieterich, J. H., 1997. Progressive failure on the North Anatolian fault since 1939 by earthquake stress triggering, Geophys. J. Int., 128(3), 594-604.

Verveer, P., 2003. GitHub repository, SciPy library.

Zebker, H., Shankar, P., \& Hooper, A., 2007. InSAR Remote Sensing Over Decorrelating Terrains: Persistent Scattering Methods, in 2007 IEEE Radar Conf., pp. 717-722, IEEE.

Zebker, H. A. \& Goldstein, R. M., 1986. Topographic mapping from interferometric synthetic aperture radar observations, J. Geophys. Res., 91(B5), 4993-4999.

Zebker, H. A., Rosen, P. A., Goldstein, R. M., Gabriel, A., \& Werner, C. L., 1994. On the derivation of coseismic displacement fields using differential radar interferometry: The Landers earthquake, J. Geophys. Res. Solid Earth, 99(B10). 
704 Zisk, S. H., 1972a. Lunar Topography: First Radar-Interferometer Measurements of 705 the Alphonsus-Ptolemaeus-Arzachel Region, Science (80-. )., 178(4064), 977-980.

706 Zisk, S. H., 1972b. A new, earth-based radar technique for the measurement of lunar 707 topography, Moon, 4(3-4), 296-306. 
Table 1: Default values for the algorithm thresholds.

\begin{tabular}{lc}
\hline Name & Value (default) \\
\hline minSize & 200 \\
$p_{\text {flux }}$ & $30 \%$ \\
$p_{m c}$ & $50 \%$ \\
$r_{m c}$ & 2 \\
\hline
\end{tabular}

Table 2: Percentage of corrected pixels for the 35 parametric tests performed on the Lebanon dataset. Column $p_{\text {flux }}>100 \%$ (respectively $p_{m c}>100 \%$ ) corresponds to step 2 (resp. step 1) only. NA: not applicable.

\begin{tabular}{|c|c|c|c|c|c|c|}
\hline$p_{p_{m c}} p_{\text {flux }}$ & $10 \%$ & $30 \%$ & $50 \%$ & $70 \%$ & $90 \%$ & $>100 \%$ \\
\hline $10 \%$ & 51.24 & 87.57 & 91.88 & 83.30 & 70.52 & 34.28 \\
\hline $30 \%$ & 87.83 & 87.49 & 90.83 & 83.16 & 40.22 & 7.89 \\
\hline $50 \%$ & 87.72 & 87.27 & 90.66 & 83.12 & 39.76 & 8.07 \\
\hline $70 \%$ & 87.36 & 86.99 & 90.52 & 83.29 & 45.66 & 13.30 \\
\hline $90 \%$ & 87.25 & 86.71 & 90.30 & 82.90 & 44.20 & 11.97 \\
\hline$>100 \%$ & 91.25 & 90.49 & 81.92 & 44.45 & 8.85 & NA \\
\hline
\end{tabular}




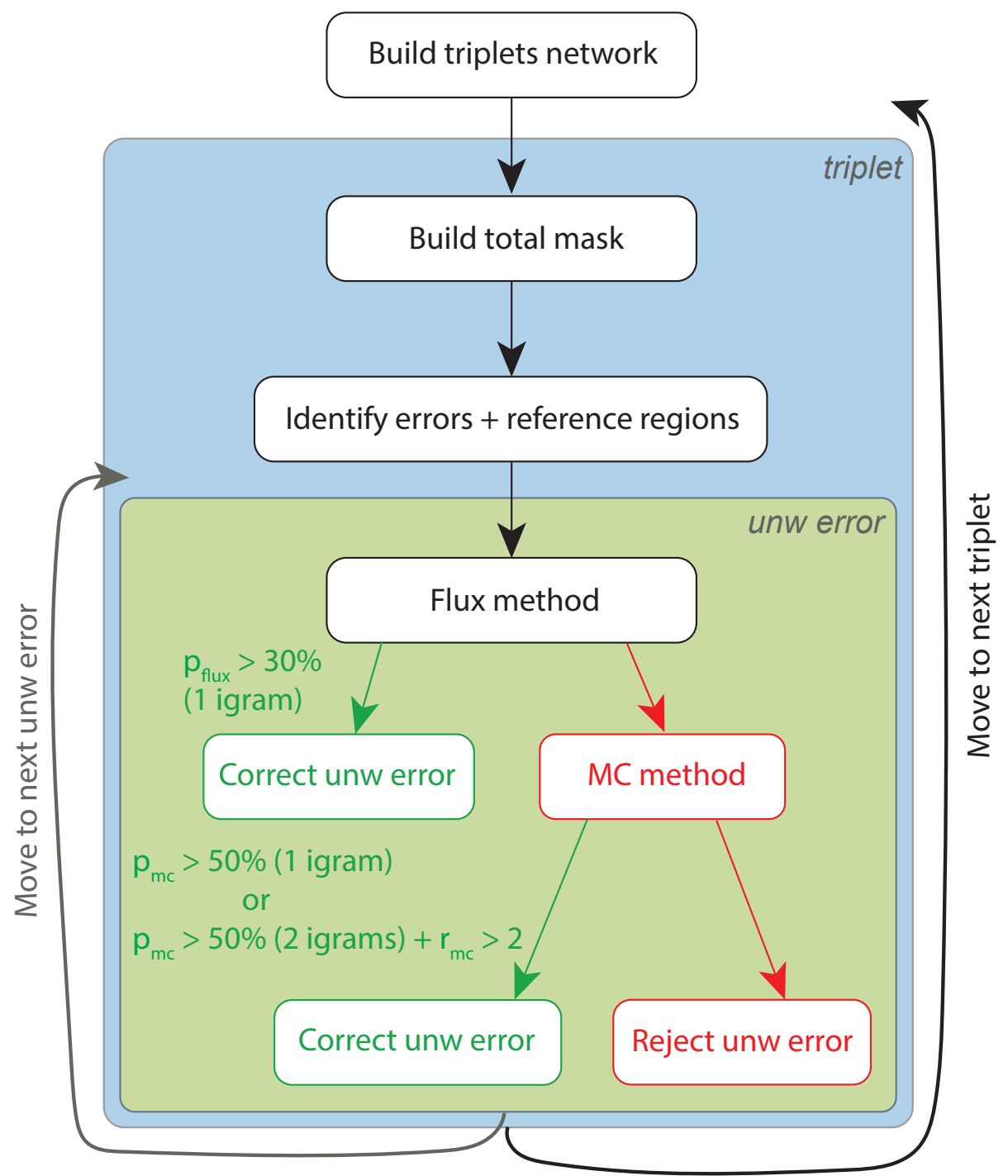

Figure 1: Algorithm implementation. First, we build the network of triplets. We process then each triplet. We identify unwrapping errors and reference regions using triplet phase closure. We correct each unwrapping error using a two-steps detection, with the flux method or with the mean closure method, in case the flux method cannot determine which interferogram to correct. 

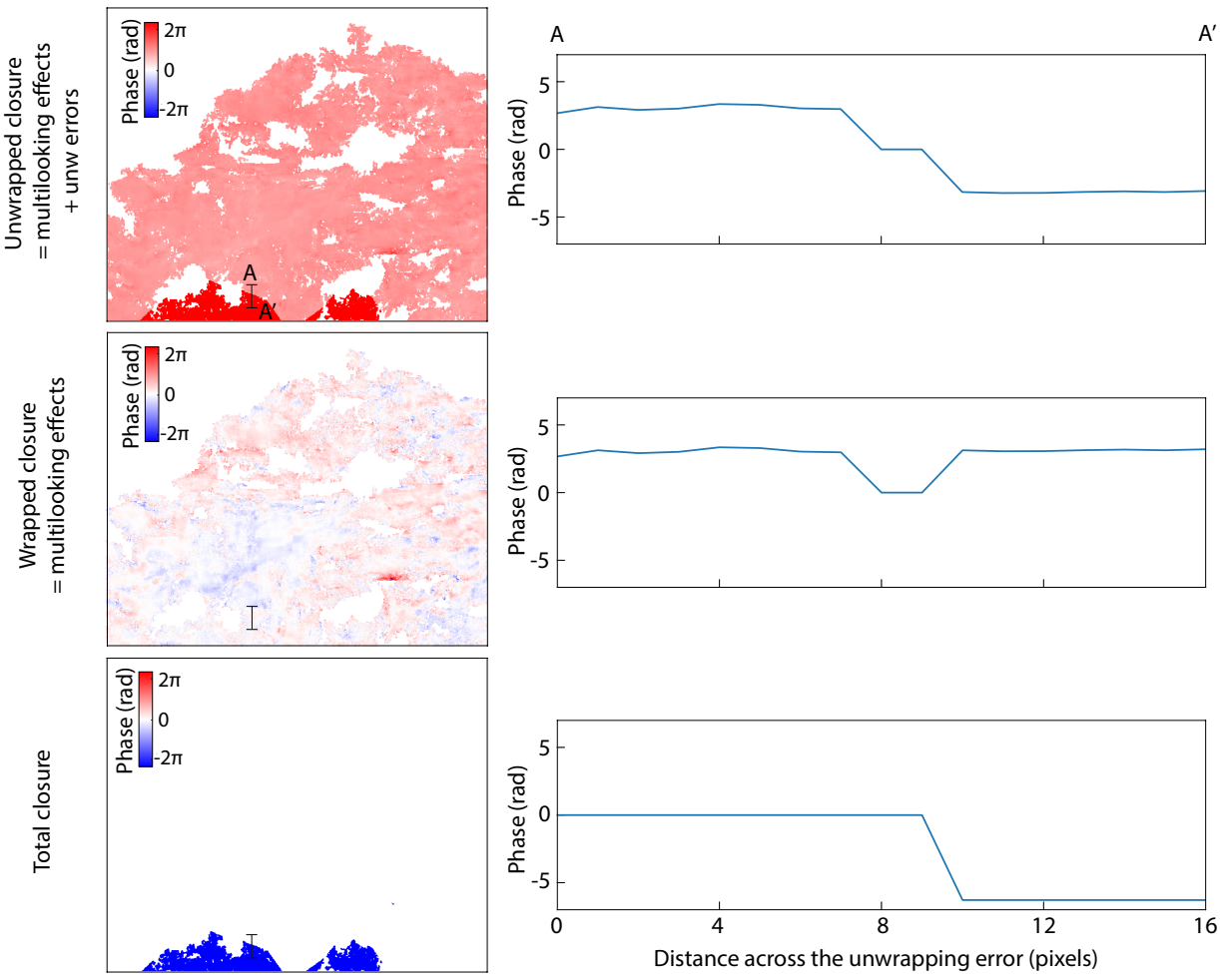

Figure 2: Closure maps (left) and profiles across an unwrapping error (right). Top) Closure from unwrapped interferograms. Center) Closure from wrapped interferograms. Non zero closure is due to multilooking. Bottom) Total closure computed by removing misclosures due to multilooking effects.
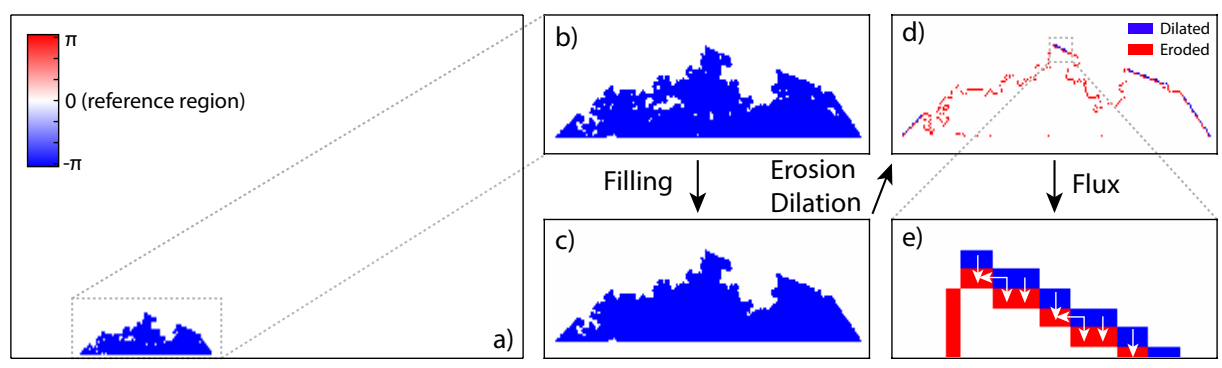

Figure 3: Steps to identify and correct an unwrapping error by the so-called flux method. a) Total phase closure of the triplet. b-c) Masked pixels within the unwrapping error zone are filled by erosion and dilation tool. d) Erosion and dilation of the unwrapping error zone to identify inner (red) and outer (blue) border. e) Computation of flux vectors between outer and inner pixels of the unwrapping error. 


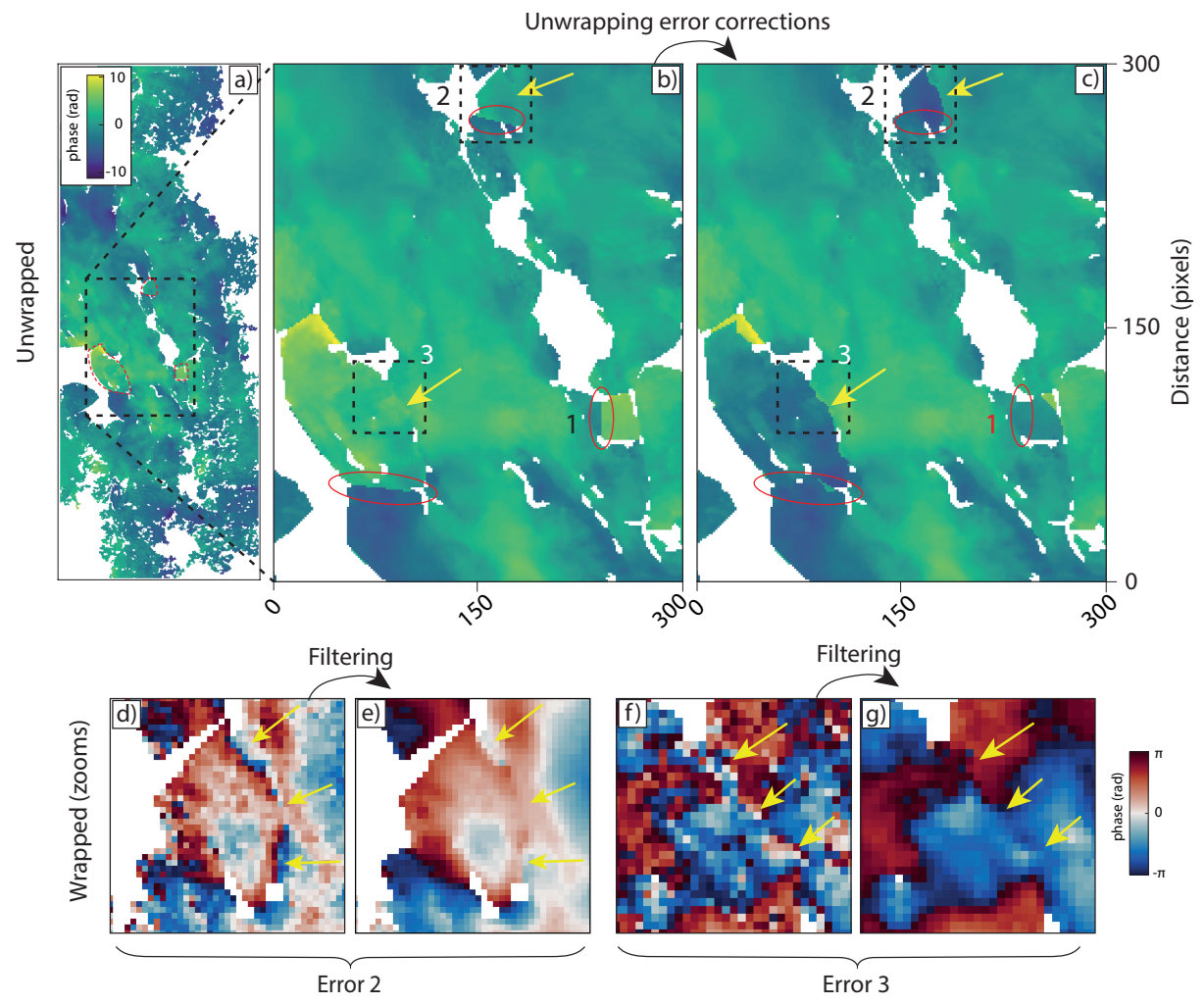

Figure 4: Results for Envisat dataset in Lebanon. a) Example of an interferogram spanning 2004/08/01 - 2008/07/06 which contains unwrapping errors (red circles). b) and c) Zooms of not corrected and corrected unwrapped interferograms. Error 1 is well corrected by the algorithm. Errors 2 and 3 are challenging areas, where the high fringe gradient, visible on wrapped interferograms, disappears by filtering before unwrapping (arrows in b). The algorithm restores the correct positions of offsets (arrows in c). d), e), f) and g) Zooms of unwrapping errors 2 and 3 on wrapped interferograms, before and after filtering. Filtering erases fringes in high fringe rate regions (arrows). 

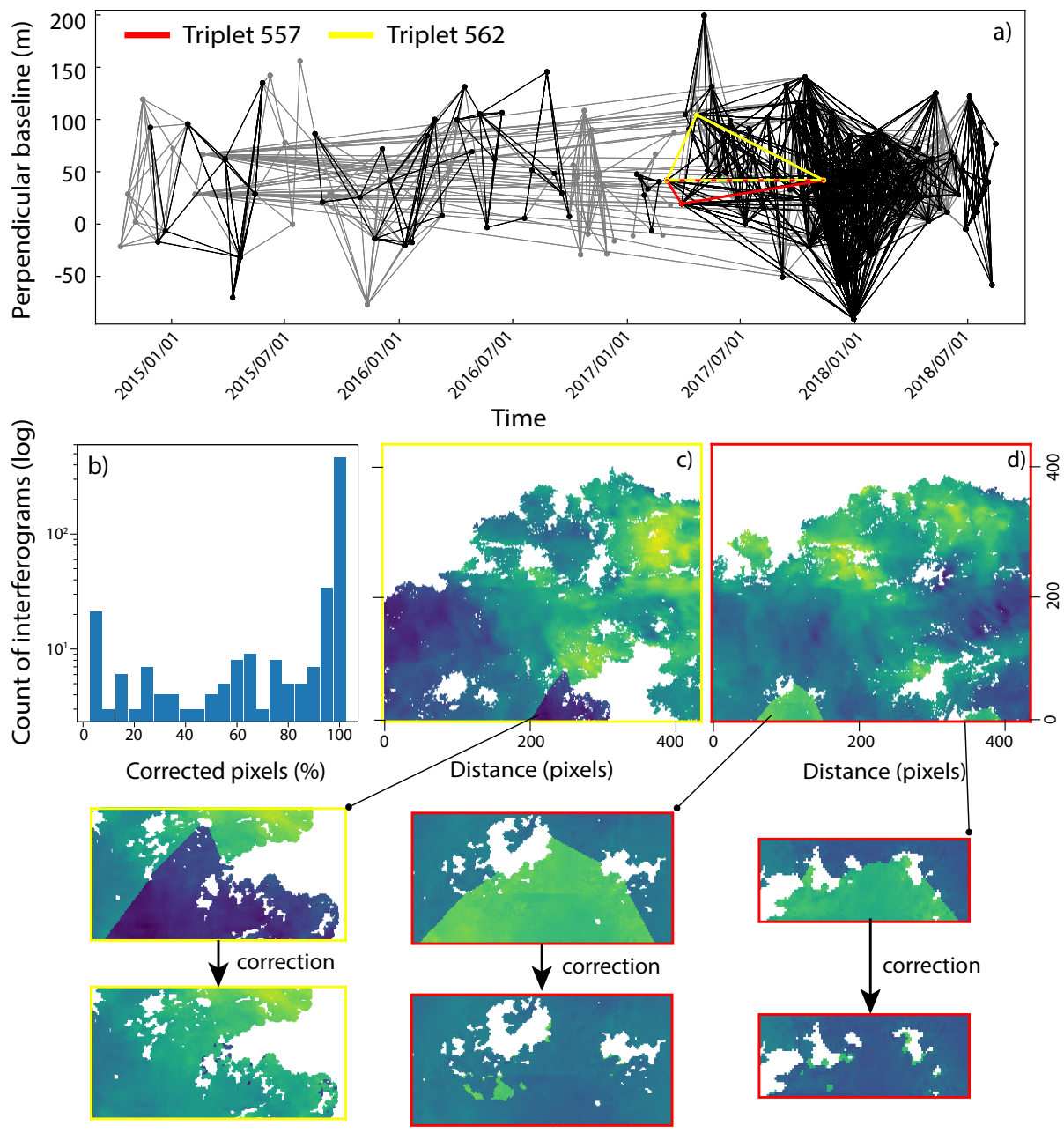

Figure 5: Results for Sentinel-1 dataset in Turkey. a) Perpendicular baseline plot with corrected triplets in black. Dots are SAR acquisitions and lines are interferograms. b) Histogram of the number of interferograms corrected as a function of percentage of corrected pixels. c) and d) Examples of corrections spanning 2017/04/22 - 2017/11/12 and 2017/03/05 - 2017/11/12, respectively. 

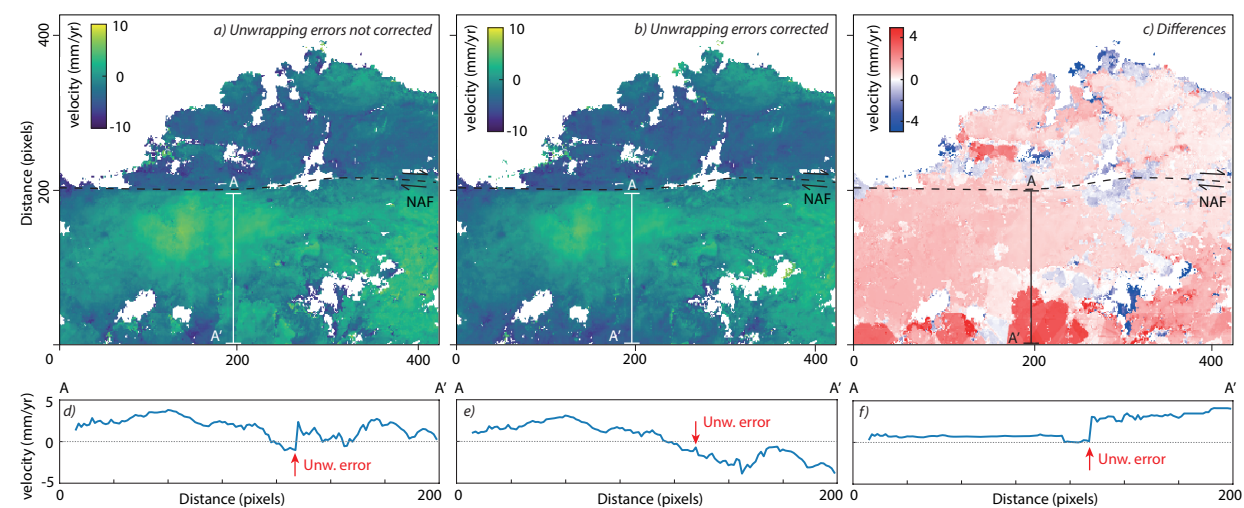

Figure 6: Influence of unwrapping error corrections on time series analysis. a) Velocity map calculated from a stack of interferograms not corrected from unwrapping errors and b) from a stack of interferograms corrected from unwrapping errors. c) Differences between a and b. d) e) and f) Profiles across a, b and c, respectively. 

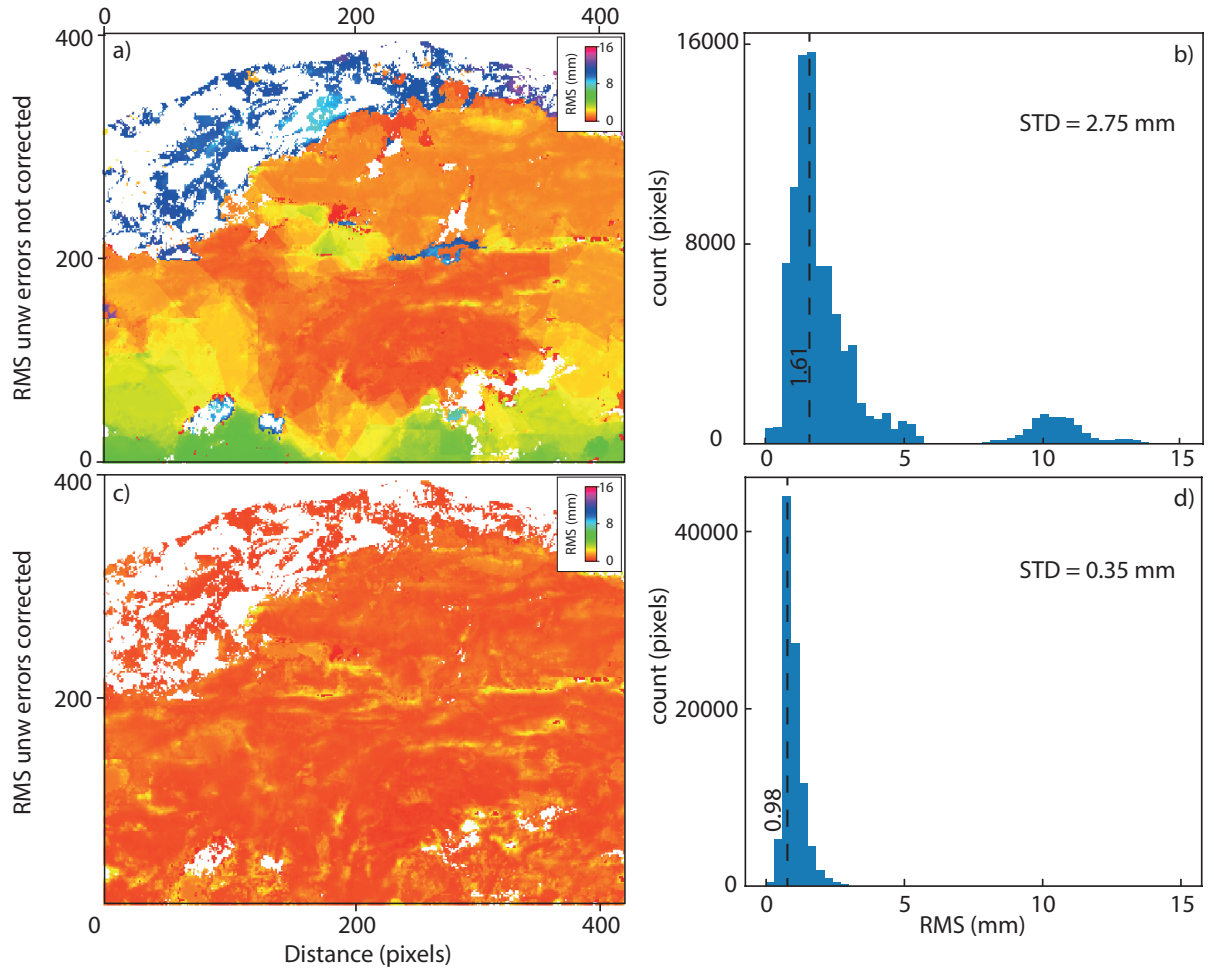

Figure 7: Influence of unwrapping errors on root mean square (RMS) maps. a) and c) RMS maps where unwrapping errors are not corrected and corrected, respectively. Unwrapping errors have a large contribution on the estimation of RMS. b) and d) Histograms of RMS maps a and c, respectively. 


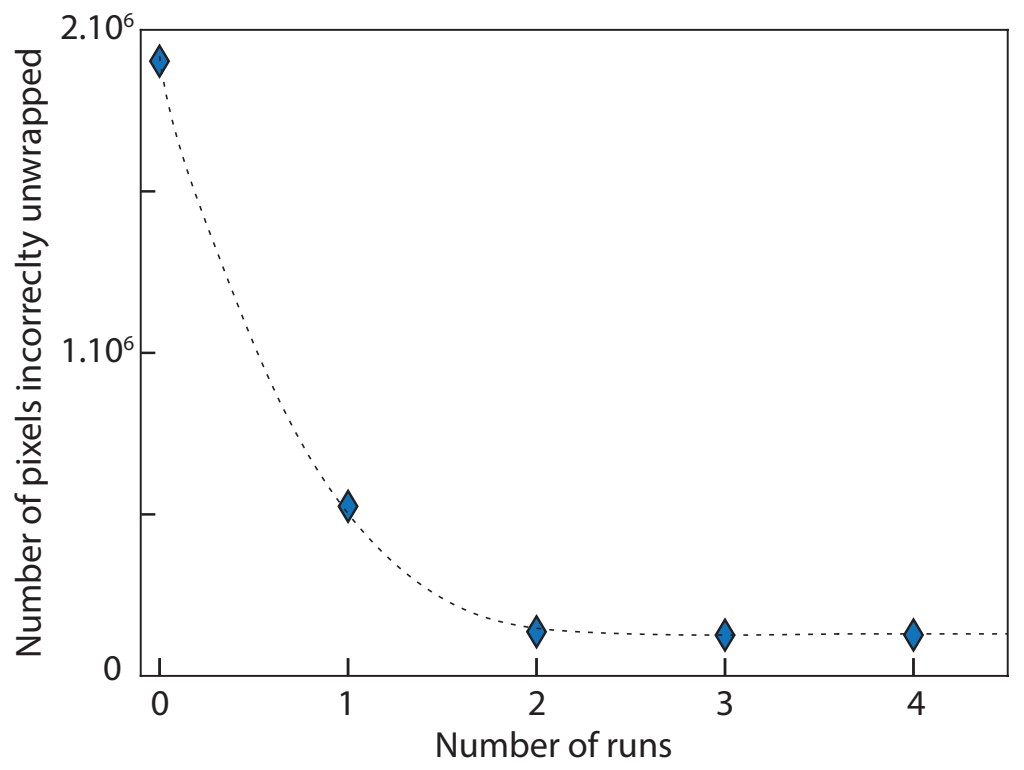

Figure 8: Number of pixels incorrectly unwrapped by a branch-cut method (unwrapping errors) detected by the algorithm during 4 successives runs. The algorithm converge in two iterations, leading to correct $95 \%$ of the 2 millions pixels incorrectly unwrapped at the beginning. 


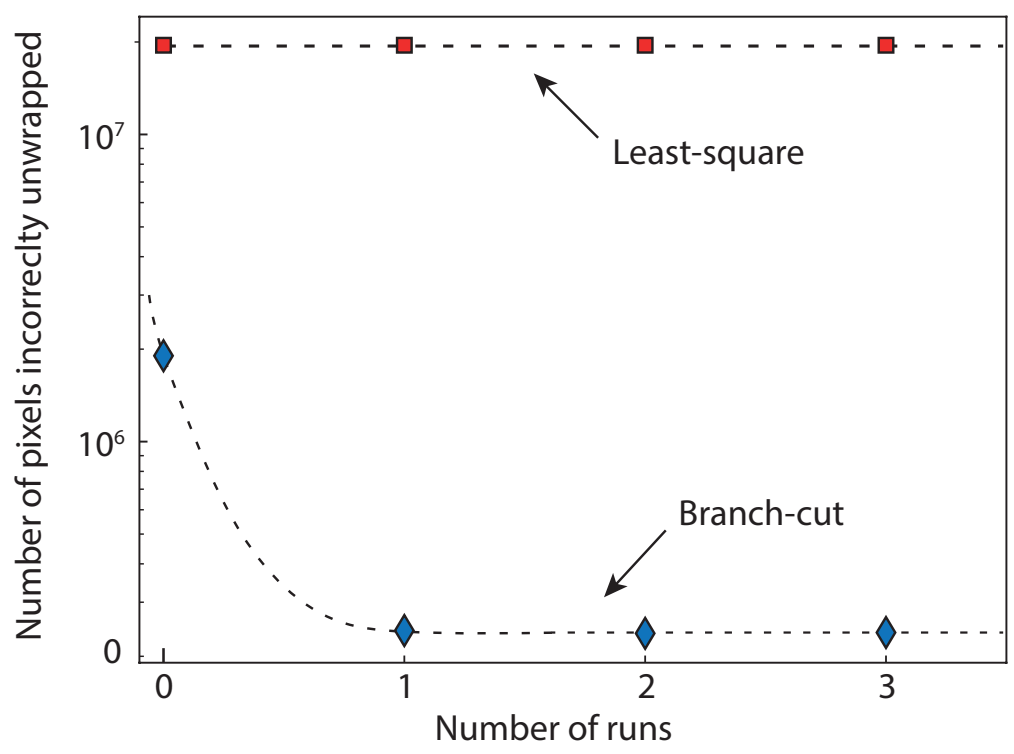

Figure 9: Comparison of the number of pixels incorrectly unwrapped by a branch-cut (blue diamonds) and a least-square (red squares) approach detected by the algorithm during 3 successives runs. 